\title{
The Application of Finite-difference Extended Kalman Filter in GPS Speed Measurement
}

\author{
Yanjie Cao ${ }^{1, a}$ \\ ${ }^{1}$ Department of Vehicle Application Engineering, Yantai Automobile Engineering Professional \\ College, City ZipCode, 265500, China \\ aemail: yanjiecao_sd@163.com
}

Keywords: Kalman filter; GPS speed measurement; Target tracking

\begin{abstract}
GPS Speed Measurement, just like position measurement, is a very widely-applied field. GPS can not only determine the real-time location of carriers in motion, but also determine the instantaneous speed of carriers . Therefore, at present, GPS speed measurement is also very widely applied. In view of the disadvantage that the present common filtering algorithm cannot track targets precisely and efficiently, a finite-difference extended Kalman filter (FDEKF) algorithm is presented to be used in GPS tracking in the reentry stage. This algorithm obtains priori and posteriori error covariance matrices using finite-difference operation, avoids nonlinear function derivation operation and the calculation of Jacobian matrix and Hessian matrix, reduces difficulty in calculation, enlarges the range of application and enhances the convergence of filtering process. The filtering accuracy is significantly improved.
\end{abstract}

\section{Introduction}

GPS speed measurement is a branch of GPS system application. When a GPS system is determining the instantaneous position of carriers, it can also determine the instantaneous speed of carriers.

The basic principle of GPS satellite positioning is to determine the point location, using ranging intersection in geomatics, i.e., to intersect the spatial position of satellite in the satellite navigation and positioning system, from more than three known ground points, and then intersect the position of unknown ground points from more than three spatial positions known to the satellite[1]. The GPS satellite launches ranging signals and navigation messages. Navigation messages contain the location information of satellite. When a ground user's GPS receiver receive signals from more than three GPS satellites at a certain moment simultaneously, it measures the distance between the observation station (the user's location) and more than three GPS satellites and solves the spatial coordinates of GPS satellites at this moment. Thereby, the location of the observation station is solved, using distance intersection.

GPS speed measurement obtains GPS signals from a GPS receiver installed on a carrier in motion and gets its speed. Although the running speeds of all carriers in motion are different, whether they are in uniform motion or not, as long as GPS signal receivers are installed on carriers, their running speed can be measured in real time. GPS receivers obtain three observed values: pseudorange, carrier phase and Doppler shift. Among them, pseudorange and carrier phase are mostly used in GPS positioning, while the observation accuracy of Doppler shift depends on the types of receivers, primarily used for speed measurement.

Currently, federated filtering algorithm and centralized filtering algorithm are commonly used in navigation. Federated filtering algorithm is used to set Kalman filter in a GPS receiver. It is a special kind of decentralized Kalman filter. Since a decentralized Kalman filter fuses positioning solutions output from a GPS receiver with DR data, it is still subject to non-model errors produced by Kalman filter in the GPS receiver[2], his paper presents finite-difference extended Kalman filter (FDEKF) algorithm, calculates the partial derivative operation of non-linear function in FDEKF algorithm using finite difference and improve the precision of GPS speed measurement 


\section{Extended Kalman Filter Algorithm}

For the trajectory target motion model in the reentry stage in this paper, define the state estimate of all measurements for the target at the moment $k$ as $X_{k / k}$, and defined the corresponding estimate error covariance matrix as $P_{k / k}$, then the one-step implementation process of extended Kalman Filter is as follows:

(1) Filter initialization. At the moment $k=0$, the state estimate of target is $X_{0 / 0}=X_{0}$. The corresponding error covariance matrix is $P_{0 / 0}=P_{0}$;

(2) The state estimate and corresponding error covariance matrix at the moment $k+1$ are

$$
\begin{aligned}
& X_{k+1 / k}=M X_{k / k}+G f_{k}\left(X_{k / k}\right)+G\left[\begin{array}{c}
0 \\
-g
\end{array}\right] \\
& P_{k+1 / k}=\left(M+G F_{k}\right) P_{k / k}\left(M+G F_{k}\right)^{T}+Q
\end{aligned}
$$

Where $F_{k}$ is the Jacobian matrix of nonlinear function $f_{k}\left(X_{k}\right)$. Take the value derived from the state estimate of the previous moment $X_{k / k}$, namely

$$
F_{k}=\left.\left[\nabla f_{k}^{T}\left(X_{k}\right)\right]^{T}\right|_{X_{k}=X_{k / k}}
$$

(3) The measurement forecast is:

$Z_{k+1 / k}=H X_{k+1 / k}$

(4) The Kalman gain is:

$$
K_{k+1}=P_{k+1 / k} H^{T}\left(H P_{k+1 / k} H^{T}+R_{k}\right)^{-1}
$$

(5) Define the state estimate and its error covariance matrix at the moment $k+1$ till all measurements at the moment $k+1$ as:

$$
\begin{aligned}
& X_{k+1 / k+1}=X_{k+1 / k}+K_{k+1}\left(Z_{k+1}-Z_{k+1 / k}\right) \\
& P_{k+1 / k+1}=\left(I-k_{k+1} H\right) P_{k+1 / k}
\end{aligned}
$$

\section{Finite-difference Extended Kalman Filter Algorithm}

The idea of finite difference was first put forward by Schei. The theoretical basis of this algorithm is to calculate the partial derivative of nonlinear functions, using the technique of polynomial approximation and first-order central difference. It has the ability of second-order nonlinear approximation. Assume that the nonlinear function $y=f(x)$, then its second-order finite central difference at $x=\bar{x}$ can be expanded as:

$$
\left.\begin{array}{l}
f(x) \approx f(\bar{x})+f_{D D}^{\prime}(\bar{x})(x-\bar{x}) \mid+\frac{f_{D D}^{\prime \prime}(\bar{x})}{2 !}(x-\bar{x})^{2} \\
f_{D D}^{\prime}(\bar{x})=\frac{f(\bar{x}+h)-f(\bar{x}-h)}{2 h} \\
f_{D D}^{\prime \prime}(\bar{x})=\frac{f(\bar{x}+h)+f(\bar{x}-h)-2 f(\bar{x})}{h^{2}}
\end{array}\right\}
$$

From Comparison Expressions (4) and (5), it can be seen that the first 3 terms in the right of Expression (5) are the same as the second-order Taylor expansion. The last 2 terms are corresponding to high-order terms. Their precisions are controlled by $h$. Obviously, the precision of expansion derived from central difference, instead of first and second-order derivatives is higher than that of general second-order Taylor series and fit for different nonlinear functions. The one-step implementation process of FDEKF is as follows[3].

(1) The filter initialization is the same as EKF. Introduce the Cholesky decomposition of the following four matrices:

$$
P_{k / k}=S_{X} S_{X}{ }^{T} ; ? P_{k+1 / k}=\bar{S}_{X} \bar{S}_{X}^{T}
$$


$Q=S_{W} S_{W}^{T} ; ? R=S_{V} S_{V}^{T}$

(2) Further state forecast:

$X_{k+1 / k}=\psi\left(X_{k / k}\right)=M X_{k / k}+G f_{k}\left(X_{k / k}\right)+G\left[\begin{array}{c}0 \\ -g\end{array}\right]$

(3) Forecast error covariance matrix:

First of all, using first-order central difference, calculate the Cholesky decomposition of forecast error covariance matrix approximately as follows:

$$
\begin{aligned}
& \bar{S}_{X}(i, j)=\left\{\frac{\psi_{i}\left(X_{k / k}+h S_{X, j}\right)-\psi_{i}\left(X_{k / k}-h S_{X, j}\right)}{2 h}\right\} \\
& j=1,2, \cdots, n_{X} \\
& P_{k+1 / k}=\bar{S}_{X} \bar{S}_{X}^{T}+Q
\end{aligned}
$$

Where $h$ is the adjustment coefficient of steps. Assume $h=3^{\frac{1}{2}}$ approximately (this is the optimal for Gaussian distribution).

(4) The measurement forecast is:

$Z_{k+1 / k}=H X_{k+1 / k}$

(5) The filter gain is:

$$
K=P_{k+1 / k} H^{T}\left(H P_{k+1 / k} H^{T}+R_{k}\right)^{-1}
$$

(1) The filter estimate and estimate error covariance matrix are:

$$
\begin{aligned}
& X_{k+1 / k+1}=X_{k+1 / k}+K\left(Z_{k+1}-Z_{k+1 / k}\right) \\
& P_{k+1 / k+1}=(I-K H) P_{k+1 / k}
\end{aligned}
$$

(6) The Choleskey decomposition of estimate error is:

$$
S_{X}(k+1 / k+1)=\left\{\operatorname{chol}\left(P_{k+1 / k+1}\right)\right\}^{T}
$$

\section{Calculation and Analysis}

The FDEKF algorithm in this paper is compared with EKF and UKF algorithms, respectively. The root-mean-square errors of their locations and speeds (represented as $E_{P}$ and $E_{V}$ respectively) are shown in Figures 1 and 2. The average root-mean-square errors (represented as $\bar{E}_{P}$ and $\bar{E}_{V}$ respectively) and the average calculation time are shown in Table 1.

Table 1. A Comparison of three algorithms in average tracking error and average calculation time

\begin{tabular}{cccc}
\hline Filter Algorithm & $\bar{E}_{P} / m$ & $\bar{E}_{V} / m \cdot s^{-1}$ & $\bar{t} / \mathrm{s}$ \\
\hline EKF & 51.3317 & 5.1947 & 0.0307 \\
UKF & 40.4431 & 3.8630 & 0.0702 \\
FDEKF & 41.1675 & 3.9899 & 0.0387 \\
\hline
\end{tabular}




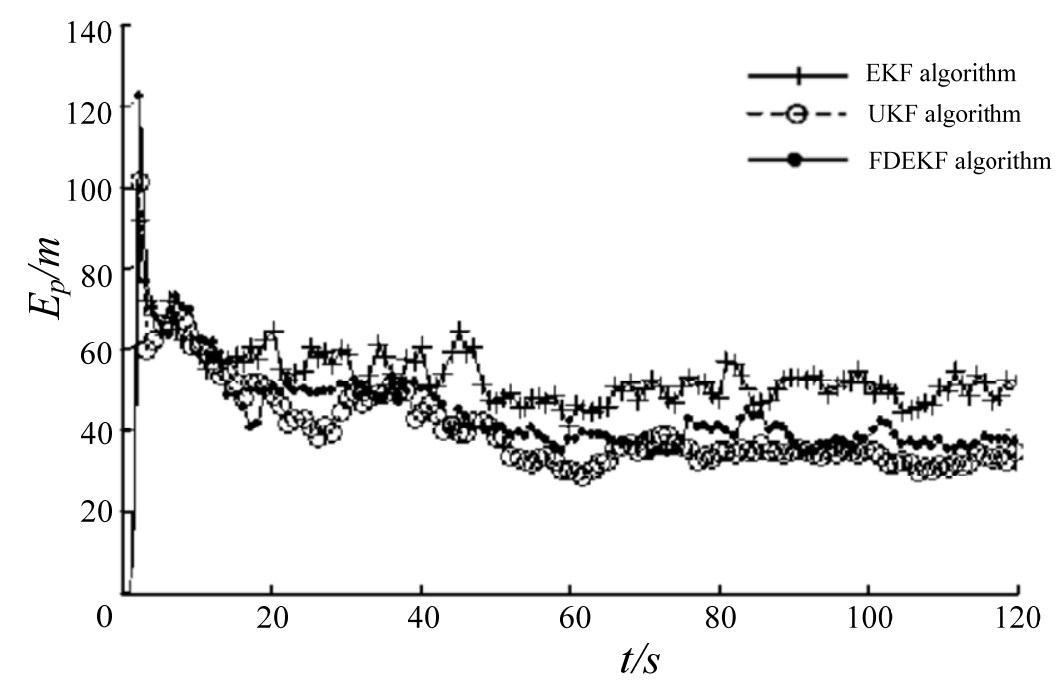

Fig.1. A comparison of three algorithms in location root-mean-square error

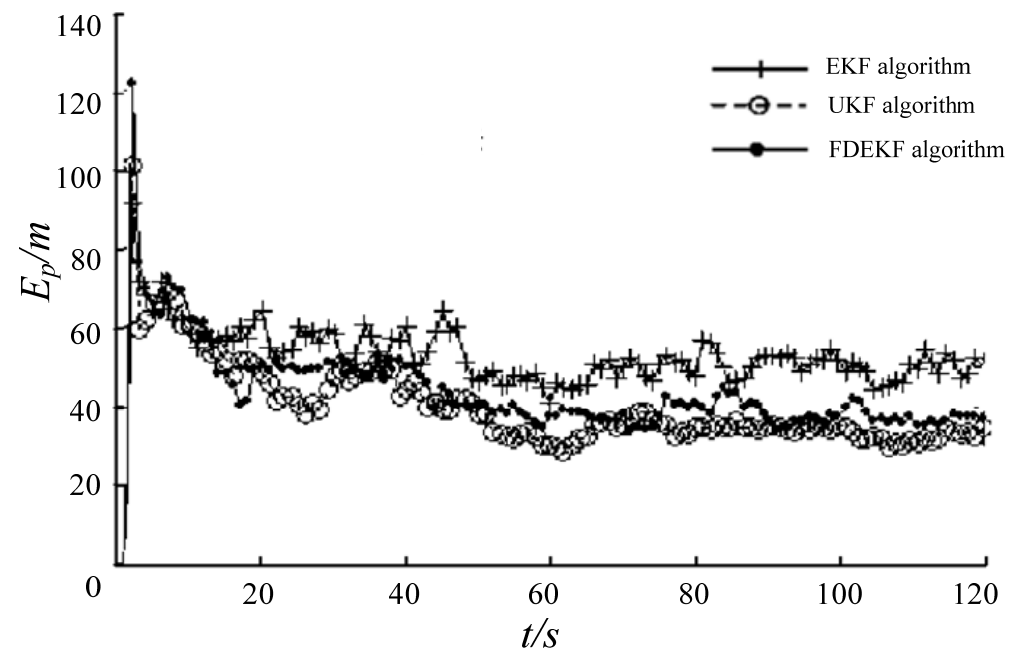

Fig.2. A Comparison of three algorithms inspeed root-mean-square error

Before performing Kalman filter, it is necessary to determine the initial value of each state parameter. Among them, the initial values of location state parameter and receiver clock error are defined as the averages of all epochs in pseudorange positioning. The derived initial values of location parameter are $(-2148744.06,4426640.72,4044655.62)$. The initial value of receiver clock error is $-73 \mathrm{~ns}$. The product of receiver clock error and speed of light is $-21.9 \mathrm{~m}$. The initial value of integer ambiguity of observation satellites is calculated based on Formula. The initial value of zenith troposphere wet delay is defined as 0 . The corresponding initial variances of state parameters are shown in Table 2. The calculation results of Kalman filter are shown in Figures 3 6.

Table 2. The initial variance of state parameter $\mathrm{m}^{2}$

\begin{tabular}{ccccccc}
\hline Name & $X$ & $Y$ & $Z$ & $\begin{array}{c}\text { Clock Error } \\
\text { Term } \\
\text { Coordinate } \\
\text { (product with } \\
\text { speed of } \\
\text { light) }\end{array}$ & $\begin{array}{c}\text { Integer } \\
\text { Ambiguity } \\
\text { ( product } \\
\text { with wave } \\
\text { length) }\end{array}$ & $\begin{array}{c}\text { Zenith } \\
\text { Troposphere } \\
\text { Wet Delay }\end{array}$ \\
\hline $\begin{array}{c}\text { Initial } \\
\text { Variance }\end{array}$ & 2500 & 2500 & 2500 & 900 & 100 & 0.25 \\
\hline
\end{tabular}




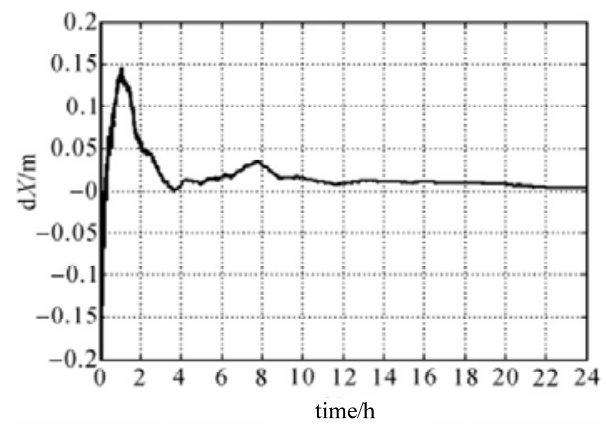

Fig.3. The difference value of $X$ coordinate

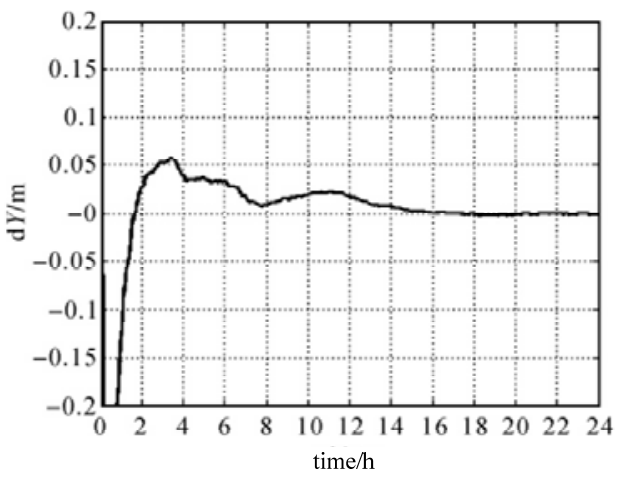

Fig.4. The difference value of $Y$ coordinate

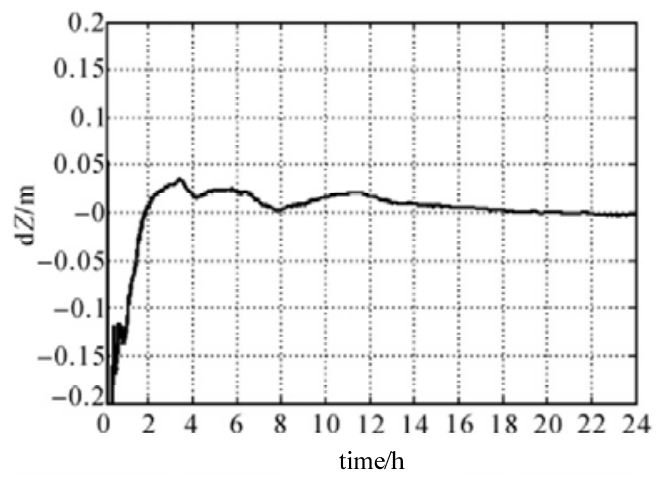

Fig.5. The difference value of $Z$ coordinate

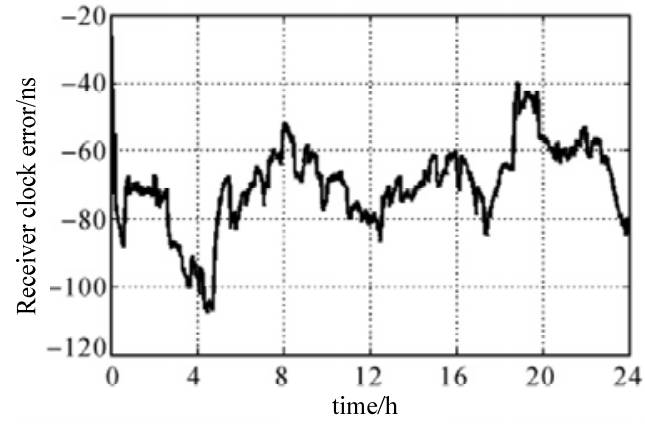

Fig.6. The receiver clock error

From Figures $3 \sim 6$, it can be seen that after Kalman filter processing, all filter values are converged within $5 \mathrm{~cm}$ in $2 \mathrm{~h}$, indicating that the program has a high convergence speed. From Figure 6, it can be seen that clock errors mostly fall into the interval between $-60 \mathrm{~ns}$ and $-80 \mathrm{~ns}$, suggesting that this receiver clock has good stability. The final convergence values of coordinates are shown in Table 3.

Table 3. Convergence values of filter

\begin{tabular}{ccccc}
\hline \multirow{2}{*}{ IGS Station } & \multirow{2}{*}{ Time } & \multicolumn{3}{c}{ Convergence Value $/ \mathrm{m}$} \\
\cline { 3 - 5 } & & $\mathrm{d} X$ & $\mathrm{~d} Y$ & $\mathrm{~d} Z$ \\
\hline BJES & $2010-04-10$ & -0.019 & -0.008 & -0.036 \\
\hline
\end{tabular}




\section{Conclusion}

Through an analysis of experimental data in this paper and calculation results of filter clock error, the clock error situation is the same as before filter. But the jump range of curve is greatly reduced, which demonstrates that this FDEKF approach is effective. It can improve the precision of GPS timing and achieve the purpose of accurate speed measurement.

In the calculation process of Kaman filter, it is necessary to pay attention to the selection of value in dynamic noise correlation matrix. If the value of $g$ is small, then the convergence speed is low. If the value of $g$ is large, then the variance of estimate error will increase. Therefore, the value of $g$ should be based on actual situation. In order to avoid filtering divergence, an adaptive filtering algorithm can be used.

\section{Reference}

[1] Luis Serrano ,Don Kin and Richad B. Langley. A Single GPS Receiver as a Real-Time Accurate Velocity and Acceleration Sensor Proceedings of ION GNSS. 2004, P 2021-2034。

[2] Jinyu $\mathrm{Hu}$ and Zhiwei Gao. Distinction immune genes of hepatitis-induced heptatocellular carcinoma[J]. Bioinformatics, 2012, 28(24): 3191-3194.

[3] Su T, Wang W, Lv Z, et al. Rapid Delaunay triangulation for randomly distributed point cloud data using adaptive Hilbert curve[J]. Computers \& Graphics, 2016, 54: 65-74.

[4] Chen Z, Huang W, Lv Z. Towards a face recognition method based on uncorrelated discriminant sparse preserving projection[J]. Multimedia Tools and Applications, 2015: 1-15. 